\title{
Checkpoint blockade in solid tumors and B-cell malignancies, with special consideration of the role of CD200
}

This article was published in the following Dove Press journal:

Cancer Management and Research

13 November 2017

Number of times this article has been viewed

\author{
Reginald M Gorczynski' \\ Fang Zhu ${ }^{2}$ \\ 'Department of Surgical Research, \\ University Health Network, \\ ${ }^{2}$ Department of Surgical Research, \\ Institute of Medical Sciences, \\ University of Toronto, Toronto, ON, \\ Canada
}

\begin{abstract}
In the ontogeny of a normal immune response, a series of checkpoints must be overcome to ensure that unwanted and/or harmful self-directed activation responses are avoided. Many of the molecules now known to be active in this overseeing of the evolving immune activation cascade, contributing inhibitory signals to dampen an overexuberant response, belong to the immunoglobulin supergene family. These include members of the CD28/CTLA-4:B7.1/ B7.2 receptor/ligand family, PD-1 and PDL-1, CD200 and CD200R, and the more recently described V-domain immunoglobulin suppressor of T-cell activation and its ligand (VSIG-3/ IGSF11). Unfortunately, from the point of view of improving immunotargeting of cancer cells, triggering these checkpoint inhibitory signaling pathways, so necessary to maintain selftolerance, simultaneously acts to prevent effective tumor immunity. The recent development of reagents, predominantly antibodies, to act as checkpoint blockade agents, has had a dramatic effect on human cancer treatment, with a marked reported success for anti-CTLA-4 and PD-1 in particular in clinical trials. This review provides a general overview of the data now available showing the promise of such treatments to our cancer armamentarium and elaborates in depth on the potential promise of what can be regarded as an underappreciated target molecule for checkpoint blockade in chronic lymphocytic leukemia and solid tumors, CD200.
\end{abstract}

Keywords: checkpoint blockade, immunotherapy, oncology, inhibitory pathways, stimulatory pathways, activated $\mathrm{T}$ cells

\section{Introduction}

The immune response to nominal antigen, including those expressed by tumor cells, involves the encounter of $\mathrm{T}$ lymphocytes with antigen expressed on suitable antigenpresenting cells (APCs), delivery of a suitable costimulatory signal (generally via the CD28:CD80/CD86 axis), and additional delivery of an activation signal to APCs. ${ }^{1}$ In the early days of studies into improving immunotherapy in cancer, a great deal of effort was spent on augmenting each of these signals, and the field is replete with studies exploring suitable mechanisms for enhanced antigen presentation and the use of costimulation for cancer therapy. ${ }^{2-4}$ However, taking as a starting point a better understanding of how and why self-recognition is controlled, in other words, how organisms ensure that there is minimal to no response to self-antigens, it soon became clear that cancer immunologists had ignored an important mechanism of immune regulation. This mechanism was associated with expression and engagement of inhibitory molecules and their receptors in the immune system..$^{5-8}$ The failure to develop autoimmune reactivity is now thought to be not simply a failure to recognize antigen in a suitable
Correspondence: Reginald M Gorczynsk Professor Emeritus, University Health Network, University of Toronto, 429 Drewry Avenue, Willowdale, M2R2K6, ON, Canada

Tel + I 6472679739

Email reg.gorczynski@utoronto.ca 
manner or the failure to express costimulatory molecules, but to the expression and functional activation of inhibitory signaling pathways controlled by inhibitory ligands/receptors, the so-called checkpoint blockade..$^{9-12}$ Thus, reversal of such checkpoint blockade, generally though not always through the use of antibodies, may release the activation of anti-tumor responses and in turn represents a major breakthrough in cancer immunotherapy. ${ }^{13-17}$

The discussion that follows highlights advances in the inhibition of checkpoint blockade in cancer care with particular attention to key molecules currently explored in both model systems and in clinical situations (see also summary of checkpoint blockade studies in Table 1 and schematic for action of reagents that target key checkpoints in tumor therapy in Figure 1).

\section{An alternate CD80/CD86 receptor, CTLA-4, as an inhibitory receptor in cancer therapy}

The early recognition that CTLA-4 represented an alternate ligand for CD80/86 but, unlike the previously identified activating ligand CD28, could produce marked effects on regulation of $\mathrm{T}$-cell responses rather than $\mathrm{T}$-cell activation led to studies exploring the effect of neutralizing CTLA-4 on immune responses in general and antitumor responses in particular. ${ }^{18-20}$ Neutralizing antibodies to CTLA-4 were the first-approved inhibitors of checkpoint blockade to be used clinically, initially in melanoma $\mathrm{a}^{21,22}$ and subsequently in other tumors (prostate/lung ${ }^{23,24}$ ). The randomized phase III study by Hodi et $\mathrm{al}^{22}$ in patients with advanced melanoma who had failed previous treatments showed for the first time that treated subjects receiving antibody (ipilimumab) alone or in combination with a gp100 peptide vaccine had superior overall survival, compared with those receiving the vaccine alone. This study represented the first positive randomized clinical trial ever reported in patients with metastatic melanoma in terms of overall survival. Subsequent studies on this promising therapy, as noted above, were extended to prostate cancer, ${ }^{24}$ initially using ipilimumab alone, but more recently using this and/or anti-PD-1 therapy (see below) in association with the first-approved cell-based immune therapy, sipuleucel-T. ${ }^{25}$ The use of inhibitors of checkpoint blockade in association with vaccine therapy is a natural extension of other studies, ${ }^{24}$ which documented that patients with advanced prostate cancer responding to the treatment with anti-CTLA-4 therapy developed enhanced responses to a number of other tumor-related antigens, both patient-specific and disease-specific, compared with nonresponders. One of these shared antigens, PAK6, was expressed in prostate cancer, was shown to induce $\mathrm{CD}^{+} \mathrm{T}$-cell responses, and was subsequently reported to be both immunogenic and protective in mouse tumor models.

Interestingly, it has been noted in patients receiving antiCTLA-4 therapy that clinical benefit may ultimately derive from treatment despite unconventional patterns of tumor response that were often, initially at least, misinterpreted as disease progression. ${ }^{26}$ For melanoma, these so-called immune-related adverse events have ranged from minor (rashes, which may rarely progress to life-threatening toxic epidermal necrolysis, and colitis, characterized by a mildto-moderate diarrhea) to more severe (lymphadenopathy, neuropathies, and nephritis). Given the previously mentioned role of CTLA-4 (and other checkpoint blockade reagents) in preventing autoimmunity, such adverse events may not be altogether surprising. At least four patterns of response have

Table I Checkpoint blockade reagents used in animal models and/or clinical situations

\begin{tabular}{lll}
\hline Target interaction $^{\mathbf{a}}$ & Checkpoint blockade reagent $^{\mathbf{b}}$ & System explored-E/C (reference) $^{\mathbf{c}}$ \\
\hline CTLA-4:CD28 & Anti-CTLA-4 & E: $(18,19,29-3 \mathrm{I})$ \\
& & C: melanoma $(22,26)$ \\
& & C: solid tumor $(24,28)$ \\
PD-I:PDL-I & Anti-PD-I & E: $(34)$ \\
& & C: melanoma $(32)$ \\
& Anti-PDL-I & C: solid tumor $(37,38)$ \\
& & E: $(35,36)$ \\
VISTA: VSIG-3/IGSFII & VISTA:KO mice & C: solid tumor (39) \\
& Anti-VISTA & E: $(49)$ \\
CD200:CD200R & Anti-CD200 & E: $(49)$ \\
& & E: hematopoietic tumor (44, 56)
\end{tabular}

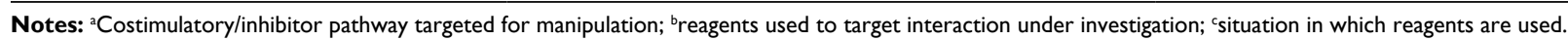
Abbreviations: C, clinical scenario; E, experimental model system; KO, knockout; VISTA, V-domain immunoglobulin suppressor of T-cell activation. 


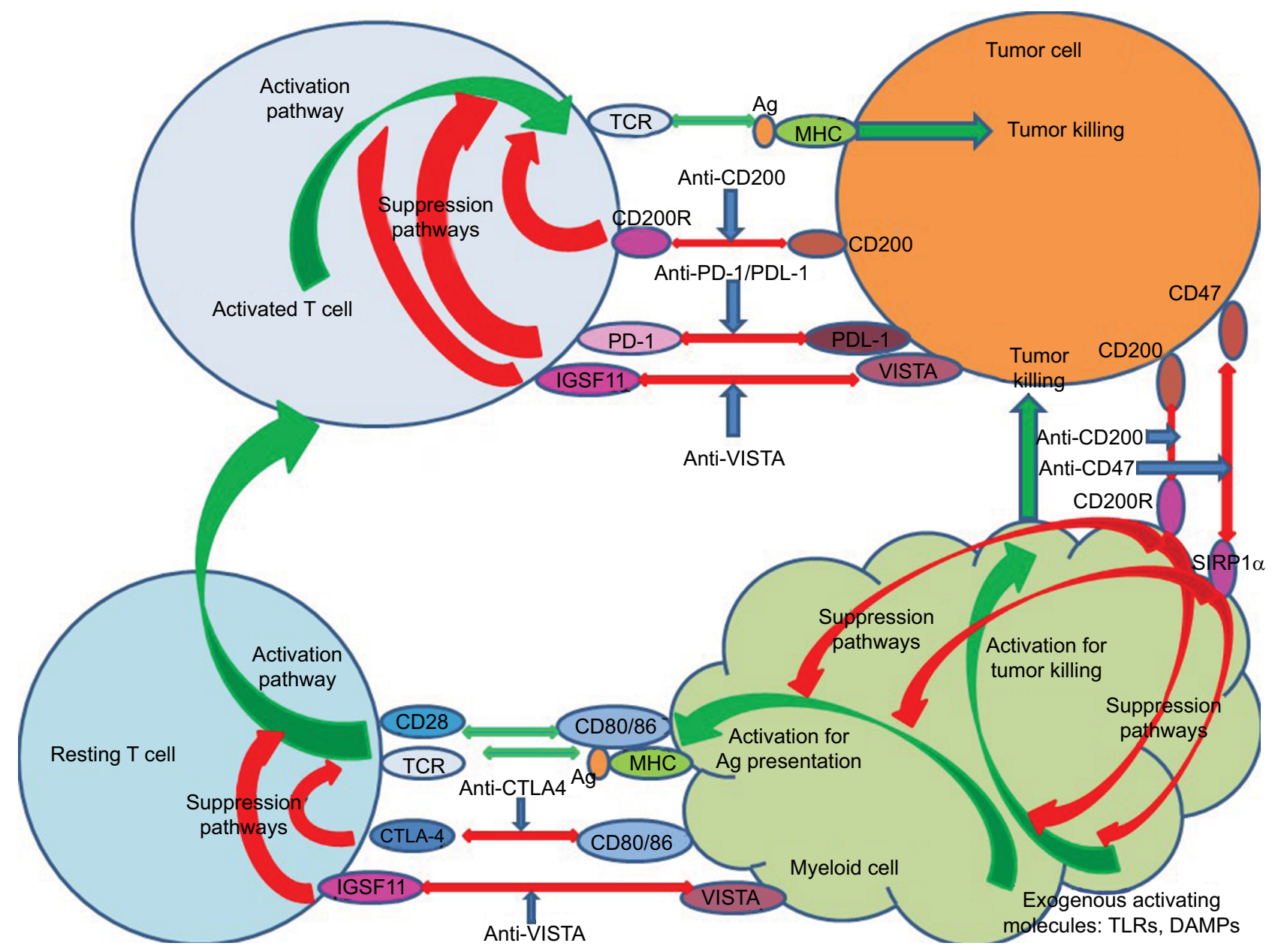

Figure I Schematic showing potential sites of action of monoclonal agents used for checkpoint blockade. Green arrows indicate activation pathways, while red indicates suppressive pathways. Blue arrows show antibodies blocking inhibitor pathways, including those determining effector pathways of tumor killing from both activated $\mathrm{T}$ cells and activated myeloid cells. Activation of resting T cells occurs following the engagement of the TCR with antigen/MHC presented by antigen-presenting cells themselves preactivated by exogenous molecules (DAMPs interacting with TLRs).

Abbreviations: Ag, antigen; DAMPs, damage-associated molecular patterns; MHC, major histocompatibility complex; TCR, T-cell receptor; TLRs, toll like receptors; VISTA, V-domain immunoglobulin suppressor of T-cell activation.

been observed in melanoma patients, namely: 1) response in baseline lesions by week 12, with no new lesions seen; 2) stable disease, followed by a slow, steady decline in total tumor burden; 3) regression of tumor after initial increase in total tumor burden; and 4) reduction in total tumor burden during or after the appearance of new lesion(s) after week $12 .{ }^{26}$ These unexpected kinetics of response to CTLA-4 treatment have in turn spurred investigation into specific approaches to evaluate responses to immunotherapy and to develop biomarkers that can predict responsiveness. ${ }^{27}$ In a recent phase I/II dose escalation/expansion study with 28 prostate cancer patients, T-cell monitoring was used in subjects receiving combined vaccination (Prostate GVAX ${ }^{\circledR}$ ) and ipilimumab immunotherapy. Differences were observed between patients who benefited from therapy and those who did not, with treatment-induced increased lymphocyte counts and $\mathrm{CD}^{+} / \mathrm{CD}^{+}{ }^{+} \mathrm{T}$-cell activation all associated with clinical benefit. Prolonged overall survival was seen in patients with the highest pretreatment frequencies of $\mathrm{CD} 4^{+} \mathrm{CTLA}-4^{+} \mathrm{T}$ cells and low pretreatment frequencies of regulatory $\mathrm{T}$ cells, suggesting that cancer-related expression of CTLA- $4^{+}$in $\mathrm{CD} 4^{+} \mathrm{T}$ cells may be a useful survival predictor and biomarker for patient selection prior to therapeutic CTLA-4 blockade in such individuals. ${ }^{28}$

A current trend has been to use anti-CTLA-4 therapy in association with other therapies, either immunotherapy (vaccination) or other checkpoint blockade reagents. In this context, the following recent studies are worthy of note. Using the nucleoside analog gemcitabine, commonly used in patients with lung, ovarian, and breast cancer, albeit with limited efficacy, Lesterhuis et al asked whether the immunopotentiating effects of this drug might normally be simultaneously, at least in part, restrained effects mediated through CTLA-4. In two independent nonimmunogenic 
murine tumor models, treatment with gemcitabine chemotherapy in combination with CTLA-4 blockade led to the induction of potent antitumor responses, mediated by both $\mathrm{CD}^{+}{ }^{+}$and $\mathrm{CD}^{+}{ }^{+}$cells. $^{29}$ In a study combining CTLA-4 blockade (ipilimumab) with VEGF blockade (bevacizumab to attenuate angiogenesis), Wu et $\mathrm{al}^{30}$ also showed evidence of increased immune cell infiltration in melanoma lesions in association with improved outcomes. More detailed studies in these treated subjects revealed an increased humoral response to Gal-1, which is known to have proangiogenesis, and immunosuppressive activity and that this increased antiGal-1 response was correlated with improved overall survival. In contrast, a subgroup of treated patients showing increased circulating Gal-1 protein had reduced overall survival. These authors concluded not only that the combination therapy might lead to improved benefit, but also that the addition of targeted therapy directed at Gal-1 might also provide adjunctive therapy, linking antiangiogenesis and immune checkpoint blockade. The results from studies by Huang et al are equally striking, in a murine model of ovarian cancer. ${ }^{31}$ These authors observed that multiple immune checkpoint molecules were expressed in tumor-associated or tumor-infiltrating cells in ovarian tumor-bearing mice and that blockade of any one of PD-1, LAG-3, or CTLA-4 alone using blocking antibodies or knockout mice led merely to a compensatory upregulation of the other checkpoint pathways. As anticipated, single-agent blockade led to tumor outgrowth in all animals, while dualantibody blockade against PD-1/CTLA-4 or triple blockade against PD-1/LAG-3/CTLA-4 led to tumor-free survival in $\sim 20 \%$ of treated mice. Dual blockade of LAG-3 and CTLA-4 pathways in PD-1 knockout mice led to tumor-free survival in $\sim 40 \%$ of treated mice, implying that a hierarchy might exist in terms of the importance of immune checkpoints. In all cases, antitumor immunity was associated with increased numbers of $\mathrm{CD}^{+} \mathrm{T}$ cells and cytokine-producing effector $\mathrm{T}$ cells, along with reduced numbers of Tregs. ${ }^{31}$

\section{Role for PD-I:PDL-I blockade in cancer therapy}

The next most prominent ligand/receptor pair investigated has been the PD-1:PDL-1 dyad, ${ }^{32}$ since earlier studies had already demonstrated that upregulation of PDL-1 was often seen on cancer cells, ${ }^{33}$ with the ligand PD-1 known to be expressed on T cells. An experimental model system used an interferon gamma (IFN $\gamma$ )-inducing cancer vaccine that included a combination of granulocyte-macrophage colony-stimulating factor and multiple toll like receptor (TLR) agonists to increase the number of activated dendritic cells (TEGVAX). It was found that while some benefit in the treatment of established tumors with TEGVAX was seen along with enhanced systemic antitumor immunity, the effect was incomplete. ${ }^{34}$ An upregulation of PDL-1 expression in the tumor microenvironment was seen in the vaccinated mice and was thought to be in part at least responsible for this incomplete tumor eradication. ${ }^{34}$ In support of this hypothesis, coadministration of a PD-1-blocking antibody with TEGVAX led to the complete regression of established tumors. In a similar manner, blockade of PDL-1, a direct target of hypoxia-inducible factor 1-alpha, led to enhanced T-cell vaccination in another animal model. ${ }^{35}$ Data from Ge et al have now also confirmed that the blockade of PDL-1 signaling augmented dendritic cell (DC) maturation, proliferation, and IL-12 secretion and led to more pronounced vaccine efficacy using DC vaccination in a breast tumor-bearing human severe combined immunodeficiency (SCID) model, with more prolonged host survival. ${ }^{36}$

Clinical trials using antibodies targeting the PD-1:PDL-1 pathway have proven to be successful in a number of malignancies. ${ }^{37}$ A phase I study reported on 39 patients with metastatic melanoma, prostate cancer, non-small-cell lung cancer (NSCLC), renal cell carcinoma (RCC), or colorectal cancer receiving a single intravenous infusion of anti-PD-1 in dose-escalating manner with those showing evidence of clinical benefit at 3 months eligible for repeated therapy. Significant effects were reported for melanoma, RCC, and NSCLC patients. A similar positive effect was reported in melanoma patients by others ${ }^{32}$ with observed responses often being long-lasting and, like the CTLA-4 story referred to earlier, often associated with cases in which progression preceded tumor shrinkage. Discrete patterns of autoimmune side effects, including vitiligo, were also often seen. As a general observation, however, most epithelial cancers have not shown responses of long duration with PD-1 or CTLA-4 blockade alone. ${ }^{32} \mathrm{~A}$ number of trials have reported positively on the use of targeting PD-1:PDL-1 with anti-PD-1 or anti-PDL-1 antibodies in association with chemotherapy on NSCLC. ${ }^{38,39}$ Other immunotherapy that might be included along with individual checkpoint blockade includes the use of other checkpoint blockade molecules (CTLA-4: above; V-domain immunoglobulin suppressor of T-cell activation (VISTA)/ Tim3/CD200: below). In addition, it is now appreciated that the analysis of biomarkers that might predict response to treatment is an important issue prior to formulation of a therapy regimen for individual patients. ${ }^{40}$

Coadministration of engineered $\mathrm{T}$ cells with chimeric antigen receptor along with immune checkpoint inhibition using PD-1 or CTLA-4 blockade has proven to be effective 
in patients with melanoma, Hodgkin's lymphoma, and NSCLC. ${ }^{41}$ In a preclinical study evaluating IL-15 (a stimulant of natural killer cells as well as $\mathrm{B} / \mathrm{T}$ cells) combined with anti-CTLA-4 or anti-PD-1 in a mouse metastatic CT26 colon carcinoma model, IL-15, though partially effective alone, increased the expression of PD- 1 on $\mathrm{CD}^{+} \mathrm{T}$ cells and increased the secretion of the immunosuppressive cytokine, IL-10. In animals receiving IL-15 with both antiPDL-1 and anti-CTLA-4, greater cytotoxic T lymphocyte killing and IFN $\gamma$ secretion were seen, along with reduced expression of PD-1 on $\mathrm{CD}^{+} \mathrm{T}$ cells and decreased IL-10 secretion, all occurring in concert with a greater survival of tumor-bearing animals, compared with mice treated with any other reagent combination. ${ }^{42}$ Again in mouse tumor models, studies have explored whether immunosuppressive cytokines might be suitable candidates for immunotherapy. Blockade of TGF- $\beta 1$ and TGF- $\beta 2$, in association with a therapeutic vaccine-inducing $\mathrm{CD}^{+} \mathrm{T}$-cell-mediated tumor immunity, led to augmented protection and increased vaccine-induced Th1-type responses as measured by IFN $\gamma$ production. ${ }^{43}$ Most importantly, when combined with PD-1 blockade, additional blockade of TGF- $\beta 1$ and TGF- $\beta 2$ led to even further increased vaccine efficacy. ${ }^{43}$

As regards biomarkers that might help delineate subjects most likely to respond to particular therapies, our own group, investigating the CD200:CD200R interaction as a checkpoint target (see below), has suggested that the presence of elevated levels of soluble CD200 in serum may reflect a poor prognosis in chronic lymphocytic leukemia (CLL) ${ }^{44,45}$ More recently, groups working on the PD-1 pathway ${ }^{46}$ have reported that elevated levels of soluble PDL-1 are associated with a poorer prognosis in $\mathrm{RCC} /$ multiple myeloma. In the case of melanoma patients, high pretreatment levels of sPDL-1 were associated with an increased likelihood of progressive disease despite treatment with CTLA-4 or PD-1 blockade, although subsequent changes in circulating sPDL-1 early after treatment were unable to distinguish responders from those with progressive disease.

\section{VISTA and other checkpoint blockade agents in immunotherapy}

VISTA has been reported to suppress T-cell responses. ${ }^{16,47}$ The molecule is predominantly expressed on hematopoietic cells and in several animal cancer models is reportedly highly expressed on myeloid cells that infiltrate tumors, although definitive identification of its ligand has yet to be performed. ${ }^{47,48}$ Recent studies investigated whether VISTA might synergize with PD-1 to produce enhanced immune regulation, using VISTA-deficient, PD-1-deficient (knockout [KO]) mice and VISTA/PD-1 double KO mice. ${ }^{49}$ Both single KO mice displayed chronic inflammation and spontaneous activation of T cells, indicating the nonredundancy of these pathways, but the double KO mice had significantly more prominent phenotypes than either of the single $\mathrm{KO}$ mice. T-cell responses to foreign antigens were observed to be higher in the VISTA/PD-1 double KO mice. Using monoclonal antibodies specific for VISTA and PDL-1, combined checkpoint blockade led to optimal tumor clearance in a cancer model, suggesting the possibility of improved clinical utility using combination therapy. ${ }^{49}$

In preliminary studies in humans, VISTA expression was measured by immunohistochemistry in a gastric cancer (GC) cohort using 464 therapy-naive GC samples and 14 samples with liver metastases. ${ }^{50}$ Tumor cell VISTA expression was seen in $\sim 9 \%$ of GC patients, and $\sim 14 \%$ of patients were found with liver metastases. VISTA expression was also observed in immune cells in $\mathrm{GC}(\sim 84 \%)$ and in those with liver metastases $(\sim 43 \%)$ although there was no correlation observed between tumor/immune cell expression and patient outcome.

Even targeting combinations of CTLA-4 and PD-1 has not to date proven to lead to durable responses in many tumors, and this in turn has fostered a search for other target molecules that might produce an adjunctive suppressive effect. Tim-3 is an inhibitory receptor expressed on many $\mathrm{T}$ cells including Foxp $3^{+}$Treg cells and innate immune cells (macrophages and dendritic cells). Engagement of its ligands leads to the suppression of cellular responses. ${ }^{51}$ In animal model systems in vivo blockade of Tim-3 with other checkpoint inhibitors led to enhanced antitumor immunity and suppression of tumor growth. ${ }^{51}$

Less studied, but potentially interesting target molecules are TIGIT and CD96 together with the costimulatory receptor CD226, which represent a pathway analogous to the CD28/ CTLA-4 pathway. ${ }^{52}$ Preliminary data already suggest that targeting these receptors can augment antitumor immune responses, although to date the immune cells affected by such treatment remain unclear. ${ }^{52}$ CD47, like CD200 that is discussed in greater depth below, is a ubiquitously expressed glycoprotein of the immunoglobulin superfamily and is thought to play an important role in self-recognition. ${ }^{53} \mathrm{~A}$ number of solid and hematologic cancers seem to exploit CD47 expression to evade immune attack, with the overexpression of CD47 correlated with poor clinical prognosis. The mechanism of action seems to depend upon interaction with SIRP $\alpha$ expressed on myeloid cells, activation of cytoplasmic immunoreceptor tyrosine-based inhibition motifs, 
recruitment of Src homology 2 domain-containing tyrosine phosphatases, and ultimately delivery of an antiphagocytic "don't eat me" signal. CD47-SIRP $\alpha$ thus seems to act as a negative checkpoint bridging innate immunity and a subsequent adaptive immune response. CD47 blocking antibodies are reported to decrease tumor growth and/or metastasis in multiple animal models. ${ }^{53}$ To date, no clinical studies targeting this pathway are available.

\section{Relevance of CD200:CD200R interactions in controlling tumor growth}

As indicated above, CD200 is relatively ubiquitously expressed, while the expression of the receptors (CD200R1-5 in mouse; CD200R1, R2 in man) is more restricted predominantly to cells of the myeloid and macrophage lineage. Early studies indicated that blockade of CD200:CD200R interactions attenuated both inflammatory (innate) immune reactions while enhancing development of acquired immunity. ${ }^{54,55}$ This in turn led to extensive investigation of the potential role of such manipulations in tumor immunotherapy.

Tumor cells in B lymphoid malignancies are known to express CD200, and thus, it was no surprise when reports of a positive effect on tumor therapy were reported for therapies aimed at neutralizing CD200 expression. ${ }^{56}$ However, the effects seen in different patients, at least in CLL, were not reflective of relative expression levels of CD200 by tumor cells, suggesting that the mechanism of action was not simply one of neutralization of expressed CD200.44,57 Wong et al reported (see also Zhou et $\mathrm{al}^{46}$ ) that soluble CD200 from serum of CLL patients was essential for the promotion of CLL growth in a NOD.SCID humanized mouse model. ${ }^{44}$ There are now reports that CD200 expression can control Treg expansion and disease progression in both acute myeloid leukemia ${ }^{58}$ and CLL. In a study with 14 CLL patients, CD200 blockade using a 1B9 antibody we described earlier ${ }^{54}$ disrupted T-cell suppression as measured in autologous mixed lymphocyte cultures (MLCs) using CD40 ligand (CD40L)-stimulated CLL cells as APCs. ${ }^{59} \mathrm{~A}$ similar mechanism was inferred by Wong et al in studies in NOD.SCID mice with CLL cells. ${ }^{44}$ Poh and Linn have suggested at least one alternative that checkpoint blockade augments cytotoxicity of cytokineinduced killer cells against human myeloid leukemia blasts. ${ }^{60}$ Even more recently, Zhu et al (personal communication) have combined a vaccination approach to CLL (using phorbol myristial acetate and ionomycin-stimulated CLLs as vaccine) in concert with blockade using 1B9 antibody, to attenuate both local disease and secondary organ spread of CLL cells in a NOD.SCID mouse model (manuscript in preparation).
The role of CD200:CD200R as a crucial checkpoint for immunity in solid tumors has also been studied. Preclinical investigations by Siva et $\mathrm{al}^{61}$ found the expression of CD200 on cell lines derived from ovarian cancer, melanoma, neuroblastoma, and RCC patients. Furthermore, adding CD200+ but not CD200- tumor cells to MLCs led to a decline in Th1 cytokine production, which was attenuated by anti-CD200 antibody. ${ }^{61}$ More recently, CD200 and CD200R protein expression was found to be increased by immunostaining on liver tissue specimens in subjects with hepatocellular carcinoma, ${ }^{62}$ compared with healthy controls. The intensity of staining with CD200R was correlated with tumor size, alpha-fetoprotein levels, and higher pathological tumor grade. Both overall and recurrencefree survival rates were lower in patients with high CD200R expression compared with those with low CD200R expression.

Attractive systems in which to study CD200 blockade are many brain tumors, which have to date been poorly responsive to tumor-derived vaccines and are known to express CD200. ${ }^{63,64}$ In one such study of glioblastoma, it was shown that glioma-derived CD200 suppressed the antitumor immune response to a vaccine. ${ }^{65}$ Using a CD200:CD200R peptide inhibitor that activates APCs, increased leukocyte infiltration into the vaccination site was seen and augmented cytokine production and antitumor cytolytic activity. Rygiel et al also reported on a role for CD200:CD200R in the regulation of skin malignancies induced in an animal model of chemical carcinogenesis. ${ }^{57} \mathrm{CD} 200 \mathrm{KO}$ mice were resistant to tumor induction, an effect correlated with an alteration in the Th17/ Treg ratio, and which occurred independent of any CD200 expression by tumor cells themselves.

Our own focus, besides the CLL studies discussed above, has concerned the role of CD200:CD200R as an important immune checkpoint in breast cancer. Interestingly, but consistent with differences observed in human disease, two quite different BALB/c mouse models have been characterized. $4 \mathrm{~T} 1$ is a highly aggressive, metastasizing, poorly immunogenic tumor, ${ }^{66}$ while EMT6 in contrast is an example of a moderately immunogenic, poorly metastasizing, and slower growing tumor. ${ }^{45}$ In the case of the former, an inflammatory cascade is growth-promoting, while for EMT6 tumors, the suppression of inflammation results in development of T-cell immunity and delayed tumor growth (see also Böger et al ${ }^{50}$ and Rygiel and Meyaard ${ }^{66}$ ). Consistent with this dichotomy, overexpression of CD200 led to improved control of growth of 4T1 tumors, while augmenting the growth of EMT6 cells. ${ }^{67}$ In contrast, mice lacking CD200 or more particularly CD200R showed markedly reduced local and metastatic EMT6 growth and could be immunized for sterile immunity with resistance 
to further tumor challenge, again with the opposite effects found for $4 \mathrm{~T} 1$ tumor. $^{68}$ Confirming the hypothesis that an active immune process was developing in EMT6 mice in association with CD200 (or CD200R) blockade, we showed that conventional chemotherapy acted synergistically with CD200 blockade to cure wild-type mice and produce immune mice resistant to rechallenge and/or metastasis even at 1 year posttreatment, an effect not seen with chemotherapy alone. ${ }^{69}$ Interestingly, although CD200 expression was detected in human breast cancer samples, no clinical data yet exist to confirm a role for anti-CD200 (or anti-CD200R) as a checkpoint in humans, although we predict that careful selection of the population (see above) will be needed to see any effects. ${ }^{70}$

\section{Summary}

Immune checkpoint blockade has unquestionably transformed the field of cancer immunotherapy producing some quite remarkable and long-lasting effects, particularly in melanoma and some hematopoietic tumors. However, it is now apparent that the overall response rate for the currently approved reagents used alone ranges only from $10 \%$ to $40 \%$. As detailed throughout this review, this has led to investigation of two additional avenues of approach: first the use of multiple checkpoint blockade reagents in combination (see Figure 1, suggesting the potential for the use of checkpoint blockade of T-cell-expressed and myeloid expressed markers) and/or with additional immunotherapy and second the analysis of biomarkers, which might predict the success of these therapies and might help monitor their efficacy. ${ }^{71}$ Development and detailed assessment of the so-far untried reagents (CD47/ CD200/VISTA) will likely also improve our armamentarium for the clinic. The other key observation of importance relates to the demographics of the at-risk population and those reflected in clinical trials. Studies of cancers diagnosed and deaths by age-group show that elderly patients ( $\geq 65$ years) are overrepresented with a further increase expected over the next decade, eg, data with NSCLC. ${ }^{72}$ Yet this population is quite underrepresented in clinical trials, and it has been known for many years that aging per se is reflected in many changes in immunity, both qualitative and quantitative. ${ }^{73,74}$ As more attention is paid to this discrepancy, we can anticipate an improved understanding of checkpoint blockade in aging, more specific clinical trials for elderly cancer patients, and an improved "juggling" of combination immunotherapies to improve cancer survival across multiple disease types.

\section{Disclosure}

The authors report no conflicts of interest in this work.

\section{References}

1. Harris NL, Ronchese F. The role of B7 costimulation in T-cell immunity. Immunol Cell Biol. 1999;77:304-311.

2. Searle PF, Young LS. Immunotherapy II: antigens, receptors and costimulation. Cancer Metastasis Rev. 1996;15:329-349.

3. Spaapen R, Blomen V, van Veelen P, et al. Identification of potential immunotherapeutic targets in antigen presentation and costimulation networks. J Immunother Cancer. 2014;2 (Suppl 3):P126.

4. Maj T, Wei S, Welling T, Zou W. T cells and costimulation in cancer. Cancer J. 2013;19:473-482.

5. Ansari MJ, Salama AD, Chitnis T, et al. The programmed death-1 (PD-1) pathway regulates autoimmune diabetes in nonobese diabetic (NOD) mice. J Exp Med. 2003;198:63-69.

6. Tivol EA, Borriello F, Schweitzer AN, Lynch WP, Bluestone JA, Sharpe AH. Loss of CTLA-4 leads to massive lymphoproliferation and fatal multiorgan tissue destruction, revealing a critical negative regulatory role of CTLA-4. Immunity. 1995;3:541-547.

7. Chambers CA, Sullivan TJ, Allison JP. Lymphoproliferation in CTLA4-deficient mice is mediated by costimulation-dependent activation of CD4+ T cells. Immunity. 1997;7:885-895.

8. Zhang J, Zhang M, Jiang W, et al. B7-H4 gene polymorphisms are associated with sporadic breast cancer in a Chinese Han population. BMC Cancer. 2009;9:394.

9. Lines JL, Pantazi E, Mak J, et al. VISTA is an immune checkpoint molecule for human T cells. Cancer Res. 2014;74:1924-1932.

10. Monney L, Sabatos CA, Gaglia JL, et al. Th1-specific cell surface protein Tim-3 regulates macrophage activation and severity of an autoimmune disease. Nature. 2002;415:536-541.

11. Nishimura H, Nose M, Hiai H, Minato N, Honjo T. Development of lupus-like autoimmune diseases by disruption of the PD-1 gene encoding an ITIM motif-carrying immunoreceptor. Immunity. 1999;11: $141-151$.

12. Okazaki T, Okazaki IM, Wang J, et al. PD-1 and LAG-3 inhibitory coreceptors act synergistically to prevent autoimmunity in mice. $J$ Exp Med. 2011;208:395-407.

13. Korman AJ, Peggs KS, Allison JP. Checkpoint blockade in cancer immunotherapy. Adv Immunol. 2006;90:297-339.

14. Wolchok JD, Yang AS, Weber JS. Immune regulatory antibodies: are they the next advance? Cancer J. 2010;16:311-317.

15. Pardoll DM. The blockade of immune checkpoints in cancer immunotherapy. Nat Rev Cancer. 2012;12:252-264.

16. Lizée G, Overwijk WW, Radvanyi L, Gao J, Sharma P, Hwu P. Harnessing the power of the immune system to target cancer. Annu Rev Med. 2013;64:71-90.

17. Ni L, Dong C. New checkpoints in cancer immunotherapy. Immunol Rev. 2017;276:52-65.

18. Chambers CA, Kuhns MS, Egen JG, Allison JP. CTLA-4-mediated inhibition in regulation of $\mathrm{T}$ cell responses: mechanisms and manipulation in tumor immunotherapy. Аппи Rev Immunol. 2001;19: $565-594$.

19. Egen JG, Kuhns MS, Allison JP. CTLA-4: new insights into its biological function and use in tumor immunotherapy. Nat Immunol. 2002;3:611-618.

20. O'Day SJ, Hamid O, Urba WJ. Targeting cytotoxic T-lymphocyte antigen-4 (CTLA-4): a novel strategy for the treatment of melanoma and other malignancies. Cancer. 2007;110:2614-2627.

21. Phan GQ, Yang JC, Sherry RM, et al. Cancer regression and autoimmunity induced by cytotoxic $\mathrm{T}$ lymphocyte-associated antigen 4 blockade in patients with metastatic melanoma. Proc Natl Acad Sci U S A. 2003; 100:8372-8377.

22. Hodi FS, O'Day SJ, McDermott DF, et al. Improved survival with ipilimumab in patients with metastatic melanoma. $N$ Engl $J$ Med. 2010;363:711-723.

23. Weber J. Immune checkpoint proteins: a new therapeutic paradigm for cancer-preclinical background: CTLA-4 and PD-1 blockade. Semin Oncol. 2010;37:430-439. 
24. Kwek SS, Dao V, Roy R, et al. Diversity of antigen-specific responses induced in vivo with CTLA-4 blockade in prostate cancer patients. J Immunol. 2012;189:3759-3766.

25. May KF Jr, Gulley JL, Drake CG, Dranoff G, Kantoff PW. Prostate cancer immunotherapy. Clin Cancer Res. 2011;17:5233-5238.

26. Weber JS, Kähler KC, Hauschild A. Management of immune-related adverse events and kinetics of response with ipilimumab. J Clin Oncol. 2012;30:2691-2697.

27. Nishino M, Ramaiya NH, Hatabu H, Hodi FS. Monitoring immunecheckpoint blockade: response evaluation and biomarker development. Nat Rev Clin Oncol. 2017;14:655-668.

28. Santegoets SJ, Stam AG, Lougheed SM, et al. T cell profiling reveals high CD4+CTLA-4 + T cell frequency as dominant predictor for survival after prostate GVAX/ipilimumab treatment. Cancer Immunol Immunother. 2013;62:245-256.

29. Lesterhuis WJ, Salmons J, Nowak AK, et al. Synergistic effect of CTLA-4 blockade and cancer chemotherapy in the induction of antitumor immunity. PLoS One. 2013;8(4):e61895.

30. Wu X, Li J, Connolly EM, et al. Combined anti-VEGF and anti-CTLA-4 therapy elicits humoral immunity to Galectin-1 which is associated with favorable clinical outcomes. Cancer Immunol Res. 2017;5:446-454.

31. Huang RY, Francois A, McGray AR, Miliotto A, Odunsi K. Compensatory upregulation of PD-1, LAG-3, and CTLA-4 limits the efficacy of single-agent checkpoint blockade in metastatic ovarian cancer. Oncoimmunology. 2016;6(1):e1249561.

32. Balar AV, Weber JS. PD-1 and PD-L1 antibodies in cancer: current status and future directions. Cancer Immunol Immunother. 2017;66:551-564.

33. Brahmer JR, Drake CG, Wollner I, et al. Phase I study of single-agent anti-programmed death-1 (MDX-1106) in refractory solid tumors: safety, clinical activity, pharmacodynamics, and immunologic correlates. J Clin Oncol. 2010;28:3167-3175.

34. Fu J, Malm IJ, Kadayakkara DK, Levitsky H, Pardoll D, Kim YJ. Preclinical evidence that PD1 blockade cooperates with cancer vaccine TEGVAX to elicit regression of established tumors. Cancer Res. 2014;74:4042-4052.

35. Noman MZ, Desantis G, Janji B, et al. PD-L1 is a novel direct target of HIF-1 $\alpha$, and its blockade under hypoxia enhanced MDSC-mediated T cell activation. $J$ Exp Med. 2014;211:781-790.

36. Ge Y, Xi H, Ju S, Zhang X. Blockade of PD-1/PD-L1 immune checkpoint during $\mathrm{DC}$ vaccination induces potent protective immunity against breast cancer in hu-SCID mice. Cancer Lett. 2013;336:253-259.

37. Topalian SL, Drake CG, Pardoll DM. Targeting the PD-1/B7-H1(PDL1) pathway to activate anti-tumor immunity. Curr Opin Immunol. 2012;24:207-212.

38. Herbst RS, Baas P, Kim DW, et al. Pembrolizumab versus docetaxel for previously treated, PD-L1-positive, advanced non-small-cell lung cancer (KEYNOTE-010): a randomised controlled trial. Lancet. 2016;387:1540-1550.

39. Rittmeyer A, Barlesi F, Waterkamp D, et al. Atezolizumab versus docetaxel in patients with previously treated nonsmall-cell lung cancer (OAK): a phase 3, open-label, multicentre randomised controlled trial. Lancet. 2017;389:255-265.

40. Tanvetyanon T, Gray JE, Antonia SJ. PD-1 checkpoint blockade alone or combined PD-1 and CTLA-4 blockade as immunotherapy for lung cancer? Expert Opin Biol Ther. 2017;17(3):305-312.

41. Hegde UP, Mukherji B. Current status of chimeric antigen receptor engineered $\mathrm{T}$ cell-based and immune checkpoint blockade-based cancer immunotherapies. Cancer Immunol Immunother. Epub 2017 May 11.

42. Yu P, Steel JC, Zhang M, Morris JC, Waldmann TA. Simultaneous blockade of multiple immune system inhibitory checkpoints enhances antitumor activity mediated by interleukin- 15 in a murine metastatic colon carcinoma model. Clin Cancer Res. 2010;16:6019-6028.

43. Terabe M, Robertson FC, Clark K, et al. Blockade of only TGF- $\beta 1$ and 2 is sufficient to enhance the efficacy of vaccine and PD- 1 checkpoint blockade immunotherapy. Oncoimmunology. 2017;6(5):e1308616.
44. Wong KK, Bannerman B, Chesny E, Spaner DD, Gorczynski RM. Soluble CD200 is critical to engraft chronic lymphocytic leukemia cells in immunocompromised mice. Cancer Res. 2012;72:4931-4943.

45. Gorczynski RM, Clark DA, Erin N, Khatri I. Role of CD200 expression in regulation of metastasis of EMT6 tumor cells in mice. Breast Cancer Res Treat. 2011;130:49-60.

46. Zhou J, Mahoney KM, Giobbie-Hurder A, et al. Soluble PD-L1 as a biomarker in malignant melanoma treated with checkpoint blockade. Cancer Immunol Res. 2017;5:480-492.

47. Dempke WCM, Fenchel K, Uciechowski P, Dale SP. Second- and thirdgeneration drugs for immuno-oncology treatment-the more the better? Eur J Cancer. 2017;74:55-72.

48. Lines JL, Sempere LF, Broughton T, Wang L, Noelle R. VISTA is a novel broad-spectrum negative checkpoint regulator for cancer immunotherapy. Cancer Immunol Res. 2014;2:510-517.

49. Liu J, Yuan Y, Chen W, et al. Immune-checkpoint proteins VISTA and PD-1 nonredundantly regulate murine T-cell responses. Proc Natl Acad Sci U S A. 2015;112:6682-6687.

50. Böger C, Behrens HM, Krüger S, Röcken C. The novel negative checkpoint regulator VISTA is expressed in gastric carcinoma and associated with PD-L1/PD-1: a future perspective for a combined gastric cancer therapy? Oncoimmunology. 2017;6(4):e1293215.

51. Das M, Zhu C, Kuchroo VK. Tim-3 and its role in regulating anti-tumor immunity. Immunol Rev. 2017;276:97-111.

52. Dougall WC, Kurtulus S, Smyth MJ, Anderson AC. TIGIT and CD96: new checkpoint receptor targets for cancer immunotherapy. Immunol Rev. 2017;276:112-120.

53. Liu X, Kwon H, Li Z, Fu YX. Is CD47 an innate immune checkpoint for tumor evasion? J Hematol Oncol. 2017;10(1):12.

54. Gorczynski RM, Chen Z, Lee L, Yu K, Hu J. Anti-CD200R ameliorates collagen-induced arthritis in mice. Clin Immunol. 2002;104:256-264.

55. Gorczynski RM. CD200 and its receptors as targets for immunoregulation. Curr Opin Investig Drugs. 2005;6:483-488.

56. Wong KK, Khatri I, Shaha S, Spaner DE, Gorczynski RM. The role of CD200 in immunity to B cell lymphoma. J Leukoc Biol. 2010; 88:361-372.

57. Rygiel TP, Karnam G, Goverse G, et al. CD200-CD200R signaling suppresses anti-tumor responses independently of CD200 expression on the tumor. Oncogene. 2012;31:2979-2988.

58. Memarian A, Nourizadeh M, Masoumi F, et al. Upregulation of CD200 is associated with Foxp3+ regulatory T cell expansion and disease progression in acute myeloid leukemia. Tumour Biol. 2013;34:531-542.

59. Pallasch CP, Ulbrich S, Brinker R, Hallek M, Uger RA, Wendtner CM. Disruption of $\mathrm{T}$ cell suppression in chronic lymphocytic leukemia by CD200 blockade. Leuk Res. 2009;33:460-464.

60. Poh SL, Linn YC. Immune checkpoint inhibitors enhance cytotoxicity of cytokine-induced killer cells against human myeloid leukaemic blasts. Cancer Immunol Immunother. 2016;65:525-536.

61. Siva A, Xin H, Qin F, Oltean D, Bowdish KS, Kretz-Rommel A. Immune modulation by melanoma and ovarian tumor cells through expression of the immunosuppressive molecule CD200. Cancer Immunol Immunother. 2008;57:987-996.

62. Sun $\mathrm{H}, \mathrm{Xu}$ J, Huang M, et al. CD200R, a co-inhibitory receptor on immune cells, predicts the prognosis of human hepatocellular carcinoma. Immunol Lett. 2016;178:105-113.

63. Moertel CL, Xia J, LaRue R, et al. CD200 in CNS tumor-induced immunosuppression: the role for CD200 pathway blockade in targeted immunotherapy. J Immunother Cancer. 2014;2(1):46.

64. Ring EK, Markert JM, Gillespie GY, Friedman GK. Checkpoint proteins in pediatric brain and extracranial solid tumors: opportunities for immunotherapy. Clin Cancer Res. 2017;23:342-350.

65. Xiong Z, Ampudia-Mesias E, Shaver R, Horbinski CM, Moertel CL, Olin MR. Tumor-derived vaccines containing CD200 inhibit immuneactivation: implications for immunotherapy. Immunotherapy. 2016;8: 1059-1071. 
66. Rygiel TP, Meyaard L. CD200R signaling in tumor tolerance and inflammation: a tricky balance. Curr Opin Immunol. 2012;24:233-238.

67. Erin N, Podnos A, Tanriover G, et al. Bidirectional effect of CD200 on breast cancer development and metastasis, with ultimate outcome determined by tumor aggressiveness and a cancer-induced inflammatory response. Oncogene. 2015;34(29):3860-3870.

68. Gorczynski RM, Chen Z, Khatri I, Podnos A, Yu K. Cure of metastatic growth of EMT6 tumor cells in mice following manipulation of CD200:CD200R signaling. Breast Cancer Res Treat. 2013;142:271-282.

69. Gorczynski RM, Chen Z, Erin N, Khatri I, Podnos A. Comparison of immunity in mice cured of primary/metastatic growth of EMT6 or 4THM breast cancer by chemotherapy or immunotherapy. PLoS One. 2014;9(11):e113597.
70. Gorczynski R, Podnos A, Kos O, Oyedele A, Zhu F, Khatri I. Significance of soluble CD200 in plasma and serum of human breast cancer patients. Int Med Rev. 2016;2(10).

71. Wang W, Liu J, He Y, McLeod HL. Prospect for immune checkpoint blockade: dynamic and comprehensive monitorings pave the way. Pharmacogenomics. 2017;18:1299-1304.

72. Elias R, Morales J, Presley C. Checkpoint Inhibitors for non-small cell lung cancer among older adults. Curr Oncol Rep. 2017;19:62.

73. Daste A, Domblides C, Gross-Goupil M, et al. Immune checkpoint inhibitors and elderly people: a review. Eur J Cancer. 2017;68:155-166.

74. Marrone KA, Forde PM. Cancer immunotherapy in older patients. Cancer J. 2017;23:219-222.
Cancer Management and Research

\section{Publish your work in this journal}

Cancer Management and Research is an international, peer-reviewed open access journal focusing on cancer research and the optimal use of preventative and integrated treatment interventions to achieve improved outcomes, enhanced survival and quality of life for the cancer patient The manuscript management system is completely online and includes

\section{Dovepress}

a very quick and fair peer-review system, which is all easy to use. Visit http://www.dovepress.com/testimonials.php to read real quotes from published authors. 